\title{
HDAC3 is essential for DNA replication in hematopoietic progenitor cells
}

\author{
Alyssa R. Summers, ${ }^{1}$ Melissa A. Fischer, ${ }^{1}$ Kristy R. Stengel, ${ }^{1}$ Yue Zhao, ${ }^{1}$ Jonathan F. Kaiser, ${ }^{1}$ \\ Christina E. Wells, ${ }^{1}$ Aubrey Hunt, ${ }^{1}$ Srividya Bhaskara, ${ }^{1}$ Jessica W. Luzwick, ${ }^{1}$ Shilpa Sampathi, ${ }^{1}$ \\ Xi Chen,, 2,3 Mary Ann Thompson, 3,4 David Cortez,, ${ }^{1,3}$ and Scott W. Hiebert ${ }^{1,3}$
}

\begin{abstract}
${ }^{1}$ Department of Biochemistry and ${ }^{2}$ Center for Quantitative Studies and Department of Biostatistics, Vanderbilt University School of Medicine, Nashville, Tennessee, USA. ${ }^{3}$ Vanderbilt-Ingram Cancer Center, Nashville, Tennessee, USA. ${ }^{4}$ Department of Pathology, Vanderbilt University School of Medicine, Nashville, Tennessee, USA.
\end{abstract}

\begin{abstract}
Histone deacetylase 3 (HDAC3) contributes to the regulation of gene expression, chromatin structure, and genomic stability. Because HDAC3 associates with oncoproteins that drive leukemia and lymphoma, we engineered a conditional deletion allele in mice to explore the physiological roles of Hdac 3 in hematopoiesis. We used the Vav-Cre transgenic allele to trigger recombination, which yielded a dramatic loss of lymphoid cells, hypocellular bone marrow, and mild anemia. Phenotypic and functional analysis suggested that $H$ dac 3 was required for the formation of the earliest lymphoid progenitor cells in the marrow, but that the marrow contained 3-5 times more multipotent progenitor cells. $\mathrm{Hdac3}^{-/}$stem cells were severely compromised in competitive bone marrow transplantation. In vitro, $\mathrm{Hdac}^{-/-}$stem and progenitor cells failed to proliferate, and most cells remained undifferentiated. Moreover, one-third of the $H d a c 3^{-/}$stem and progenitor cells were in $\mathrm{S}$ phase 2 hours after BrdU labeling in vivo, suggesting that these cells were impaired in transit through the $S$ phase. DNA fiber-labeling experiments indicated that $\mathrm{Hdac} 3$ was required for efficient DNA replication in hematopoietic stem and progenitor cells. Thus, $\mathrm{Hdac} 3$ is required for the passage of hematopoietic stem/progenitor cells through the $S$ phase, for stem cell functions, and for lymphopoiesis.
\end{abstract}

\section{Introduction}

Chromatin-modifying enzymes have emerged as useful targets for pharmacological inhibition in a broad range of diseases from neurological disorders to cancer. Inhibiting these enzymes can be used to increase or decrease gene expression in a tissue-specific fashion, which may have special utility in diseases where increasing the dosage of a gene has dramatic long-term benefits (e.g., Friedreich ataxia; refs. 1-4). In cancer, hematopoietic malignancies represent an especially attractive target for epigenetic therapy, as histone methyltransferases and histone acetyltransferases are directly involved in some forms of leukemia (e.g., acute leukemia associated with chromosomal translocations of MLL and/or CBP). Moreover, histone deacetylases are recruited by oncoproteins in some leukemias and subtypes of B cell lymphoma (5-8). In addition to affecting gene expression, chromatin must be opened during DNA replication and re-compacted thereafter, which provides opportunities to cause replication fork stalling and DNA damage that will kill inappropriately cycling cancer cells.

Histone deacetylase (HDAC) inhibitors were initially found to be active against hematopoietic malignancies due to their ability to trigger differentiation of a variety of myeloid leukemia cell lines (9-11). Two of these compounds (SAHA or vorinostat, and depsipeptide or romidepsin) have gained FDA approval for their use against cutaneous $T$ cell lymphoma, and these and other broad-spectrum inhibitors (e.g., SAHA targets HDAC1, 2, 3, 6, and $8)$ are in various stages of clinical trials for both solid tumors and hematopoietic malignancies $(12,13)$. However, the mechanism of

Authorship note: Alyssa R. Summers, Melissa A. Fischer, and Kristy R. Stengel contributed equally to this work.

Conflict of interest: The authors have declared that no conflict of interest exists. Citation for this article: J Clin Invest. 2013;123(7):3112-3123. doi:10.1172/JCI60806 action is still not fully elucidated, and the HDAC(s) responsible for their activity is still not clear. Gene targeting in mice has provided key information on the physiological roles of individual HDACs and how they contribute to the control of chromatin structure, gene expression, and cellular differentiation programs. For example, Hdac1 and Hdac2 appear to work together biochemically, but deletion of Hdac1 or Hdac2 yielded distinct phenotypes, even though there was some compensation by the other family member during development (14-17). However, the double deletion of these genes dramatically impaired proliferation in multiple cell types by blocking the G1/S phase transition (18-22).

Histone deacetylase 3 (HDAC3) is targeted to differing degrees by all of the commonly used histone deacetylase inhibitors and plays a key role in regulating site-specific transcription $(23,24)$. Hdac3 was initially found in homology searches using the other class 1 HDACs and has been largely studied as a component of the NCOR/SMRT repression complex that is recruited by nuclear hormone receptors and other site-specific DNA binding factors to repress the expression of individual genes (25-30). Because of the role of nuclear hormone receptors (such as the retinoic acid receptor) in hematopoiesis, $H d a c 3$ may be a target for the effects of HDAC inhibitors on differentiation. Intriguingly, gene deletion in mice has demonstrated that Hdac 3 also contributes to the control of global levels of histone acetylation that regulate chromatin structure. Cells lacking Hdac3 showed increases in H4K5ac, $\mathrm{H} 4 \mathrm{~K} 12 \mathrm{ac}$, and in some cases $\mathrm{H} 3 \mathrm{~K} 9 / \mathrm{K} 14 \mathrm{ac}$, which led to a loss of heterochromatin, an increase in DNA double-strand breaks, and affected proliferation $(31,32)$. This led to embryonic lethality, but deletion in adult tissues was surprisingly tolerated (33-37). Nevertheless, deletion of Hdac3 in the heart or the liver caused hypertrophy that was accompanied by altered metabolism $(34,37)$. When deleted in the liver, Hdac3 inactivation caused a fatty liver pheno- 
A
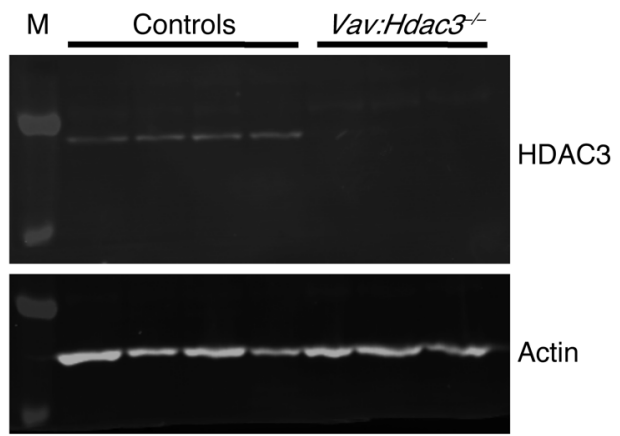

B

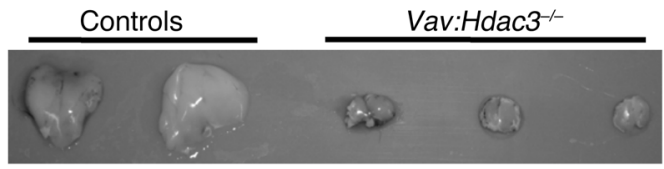

C
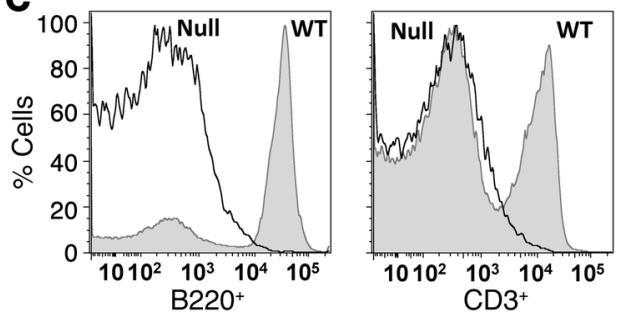

D

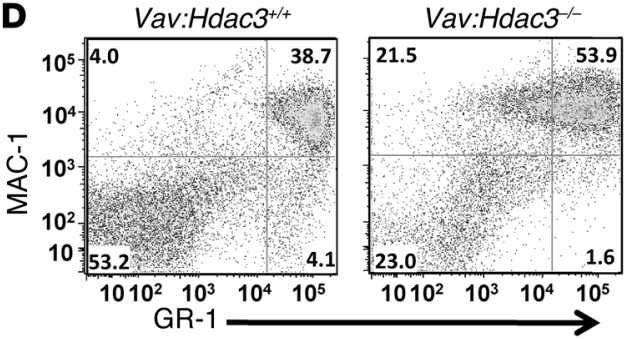

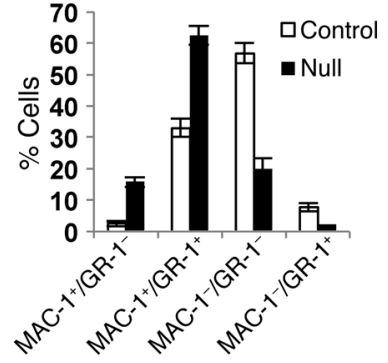

Figure 1

Hdac3 is required for lymphopoiesis. (A) Western blot of HDAC3 expression in total bone marrow. (B) Gross morphology of thymus from 6-week-old mice. (C) FACS analysis of B220 and CD3 expression in spleens. (D) Analysis of myelopoiesis in total bone marrow samples using antiMAC-1 and anti-GR-1. Numbers in each box represent the relative percentage of the cells in the indicated gated population. All FACS plots shown are representative of at least 5 mice. Graph at the right is a summation of biological replicates using 4 WT and 6 null mice first gated on GFP+ cells (MAC-1+/GR-1-, $P=0.002$; MAC-1+/GR-1 ${ }^{+}, P=0.007$; MAC-1-/GR-1-, $P=0.005$; MAC-1-/GR-1 $\left.{ }^{+}, P=0.008\right)$.

it may be a major target of HDAC inhibitors, which affect DNA replication (40), further suggesting that pharmacological inhibition of HDAC3 may be beneficial in hematopoietic malignancies.

\section{Results}

Hdac3 is essential for lymphoid commitment. We crossed our Hdac3 floxed mice with Vav-Cre transgenic mice to examine the effects of disrupting this key regulator of chromatin structures in HSCs. The Vav promoter is expressed during late embryogenesis and primarily targets hematopoietic lineages, including the stem cells, but also affects endothelial cells (38). Overall, Vav:Hdac3 $3^{-/}$mice were obtained at the expected frequencies, but we noted that half of these mice showed signs of anemia (e.g., pale paws) (Supplemental Figure 1A; supplemental material available online with this article; doi:10.1172/ JCI60806DS1), and some failed to survive past 4-5 weeks of age. Western blot analysis of Hdac 3 expression in the bone marrow of those Vav:Hdac3-mice that survived to 6 to 8 weeks of age demonstrated that Vav-Cre was very efficient at deleting Hdac3 (Figure 1A). However, to ensure that we

type, as well as disruptions in circadian rhythms $(33,35)$. When changes in gene expression and metabolism were coupled with a loss of control of chromatin structures and the inherent genomic instability caused by altered chromatin, inactivation of Hdac3 led to hepatocellular carcinoma (32).

Classically, hematopoietic stem cells (HSCs) have been used to define the role of transcription factors and chromatin-modifying factors in adult stem cell functions, lineage allocation, and differentiation. This is largely due to the availability of functional assays for stem cell activities as well as immunophenotyping and other in vitro assessments of HSC and progenitor cell functions. To begin to understand how Hdac3 might contribute to the function of adult stem cells, we deleted this gene throughout hematopoiesis using the Vav-Cre transgene (38). These mice were viable but had a dramatic loss of $\mathrm{T}$ and $\mathrm{B}$ cells. Immunophenotyping traced this defect to a loss of the earliest lymphoid progenitor cell that is $\mathrm{LIN}^{-}$ $/ \mathrm{SCA}^{+} / \mathrm{c}^{-\mathrm{KIT}^{+}} / \mathrm{FLT}^{\text {hi }}$ (39), with an accumulation of multipotent progenitor (MPP) cells. In addition, the loss of Hdac3 crippled stem and progenitor cell proliferation, causing aberrant cycling of the multipotent populations, which coincided with defects in DNA replication. Thus, Hdac3 is essential for stem cell functions and for the production of the earliest lymphoid progenitor cells, and always assessed only Hdac3-deficient cells, we crossed our mice with ROSA26-Lox-Stop-Lox-GFP transgenic mice such that cells expressing Cre to delete Hdac3 also expressed GFP (41). FACS for GFP confirmed a greater than $85 \%$ deletion efficiency (Supplemental Figure 1B). Upon necropsy of these mice, we noted that the size of the thymus in the Vav:Hdac3 $3^{-/}$mice was severely reduced compared with the littermate control mice (Figure 1B). The thymus from the null mice typically contained only $1 \%$ of the normal complement of T cells (Supplemental Figure 1C). By contrast, the spleens in the Vav:Hdac3 $3^{-/}$mice were relatively normal in size, but FACS analysis showed a dramatic loss of $\mathrm{CD}^{+} \mathrm{T}$ cells, as well as a dramatic decrease in B220+ B cells (Figure 1C). Contrary to containing mostly lymphoid cells, the spleens were largely composed of myeloid cells and stroma (data not shown).

Given the dramatic loss of B and T cells in the peripheral organs and blood (Figure 1 and data not shown), we examined the bone marrow. The marrow from Vav:Hdac $3^{-/}$mice was hypocellular, with the total number of cells reduced by half (Supplemental Figure 1D). While the bone marrow contained fewer cells, the lineage-negative $\left(\mathrm{LIN}^{-}\right)$fraction was increased by roughly 3 fold (Supplemental Figure 1D). Since there are few T cells in the bone marrow, we examined B cells. Consistent with the lack of B220 

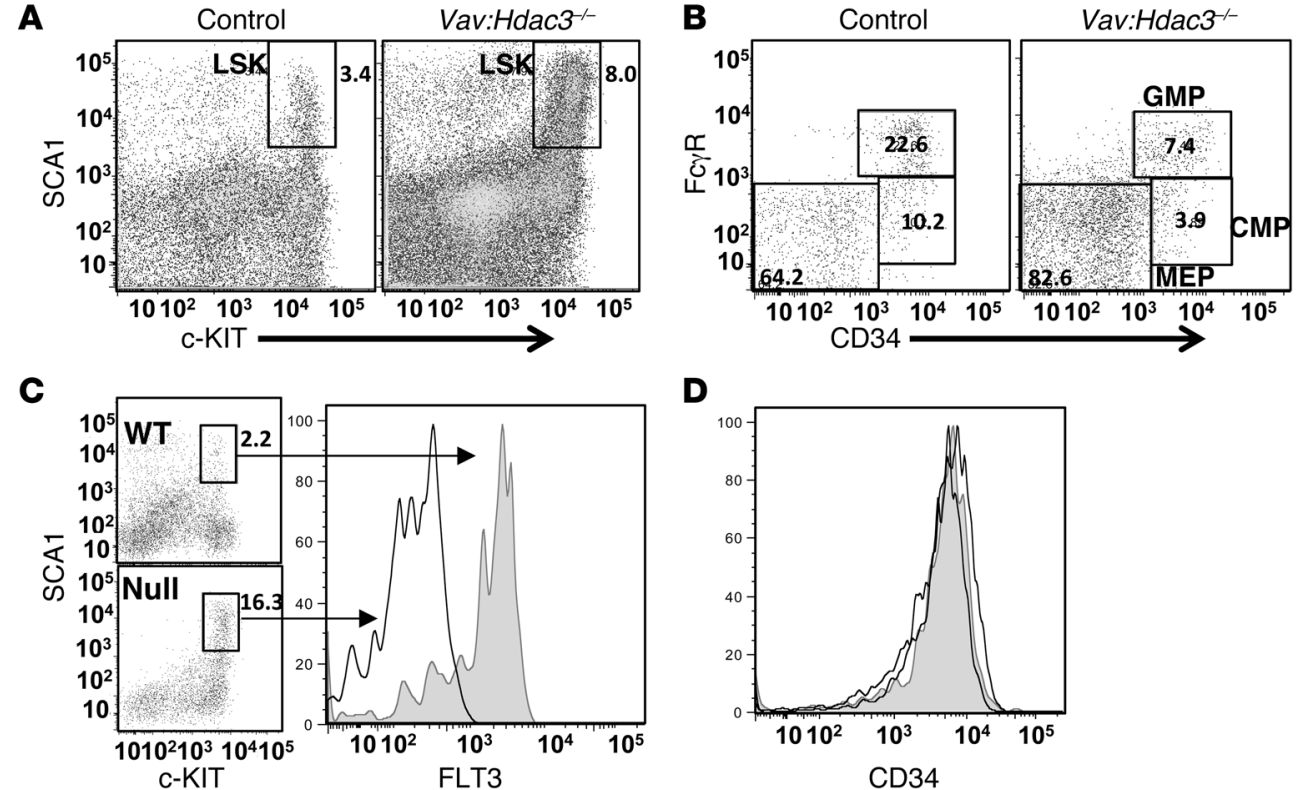

D

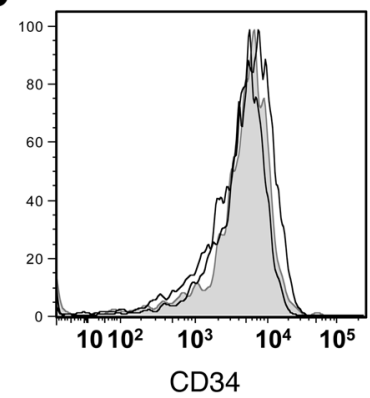

$\mathbf{E}$
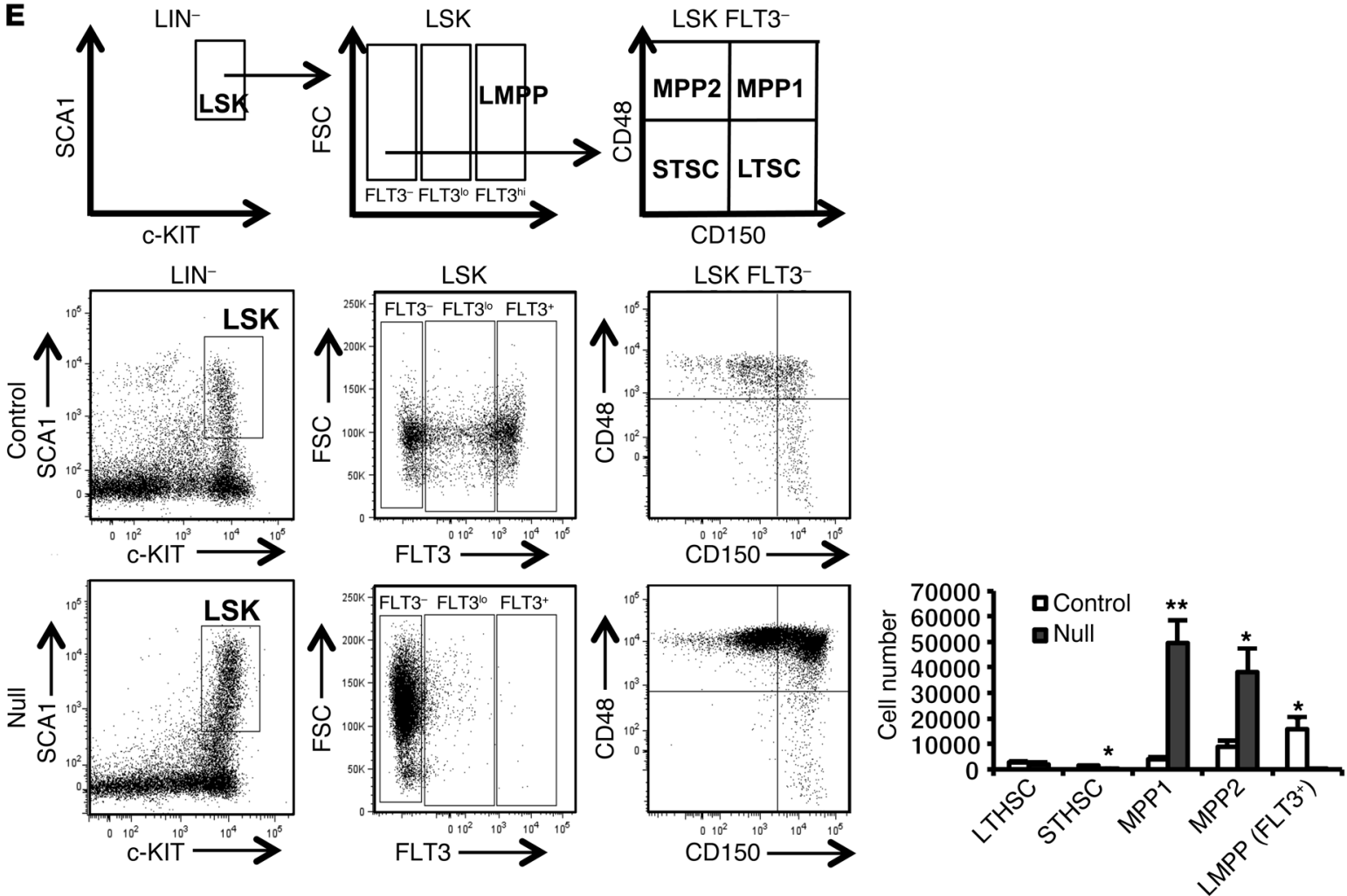

Figure 2

Inactivation of $\mathrm{Hdac} 3$ increases early stem and progenitor cells and blocks progression to the $\mathrm{FLT3}^{+}$stage. FACS analysis of LIN- bone marrow to examine the (A) LSK population using SCA1 and c-KIT antibodies. (B) FACS analysis of LIN- and c-KIT+ cells in A using Fc $\gamma R$ and CD34 antibodies to distinguish GMP, CMP, and MEP populations. (C) Further analysis of LSK population (small plots on the left) using FLT3 antibody and (D) CD34 antibody. Representative FACS plots from at least 5 mice are shown. In D, the shaded curves designate the control mice and the open curves are the null mice. (E) Immunophenotypic analysis of stem and MPP cells. Top panels show the scheme for the flow cytometric analysis and the markers used. Graph at right shows the actual number of cells per leg (STHSC, $P=0.0375$; MPP1, $P=0.0069$; MPP2, $P=0.04$; LMPP, $P=0.0295)$. Numbers in each box indicate the relative percentage of the cells in the indicated gated population. ${ }^{*} P<0.05 ;{ }^{* \star} P<0.007$. LTHSC, long-term HSC; STHSC, short-term HSC. 
A

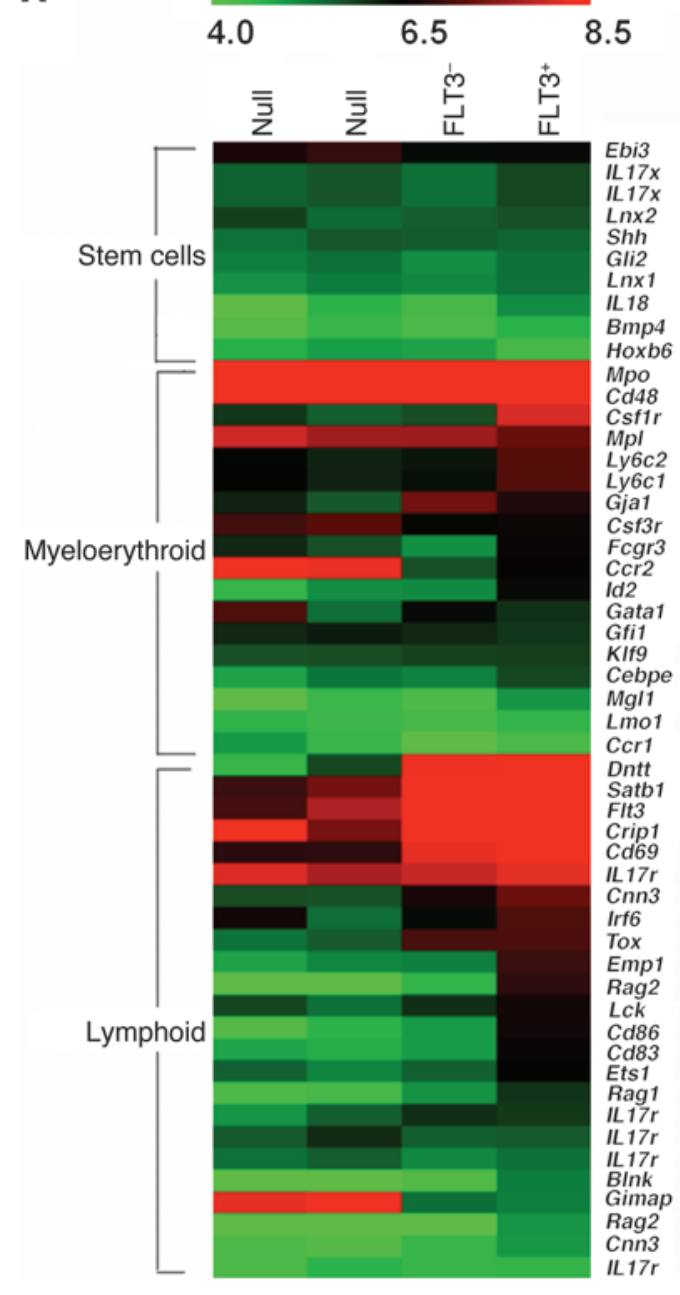

B

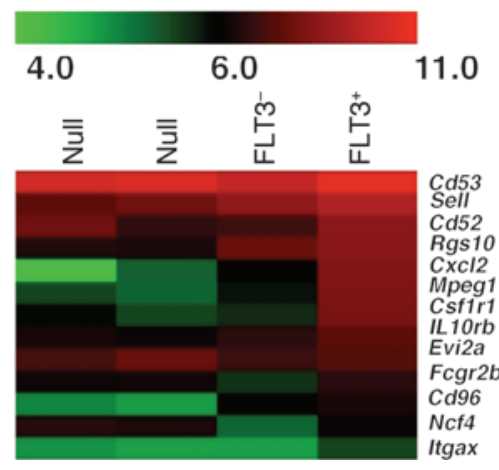

$\mathrm{B}$ cells in the spleen, there was a dramatic loss of B cells in the bone marrow (Supplemental Figure 1E). The myeloid compartment showed an increase in the relative percentage of GR-1/MAC-1 double-positive cells, a somewhat lower percentage of GR-1+/ MAC- $1^{\text {lo }}$ cells, and a subtle increase in the relative percentage of $\mathrm{MAC}-1^{+} / \mathrm{GR}-1^{\text {lo }}$ (Figure $\left.1 \mathrm{D}\right)$. When the MAC- $1^{+}$cells were further fractionated using anti-Ly6C and Ly6G, we noted an increase in the relative levels of Ly $6 \mathrm{G}^{\mathrm{lo}} / \mathrm{Ly} 6 \mathrm{C}^{\mathrm{lo}}$ cells, with a concomitant drop in the percentage of Ly6G ${ }^{\text {lo }} /$ Ly6 $\mathrm{C}^{\text {hi }}$ cells (Supplemental Figure 1G and ref. 42). The null animals had similar relative levels of Ter119+

\section{Figure 3}

Hdac3 is required for lymphoid priming of bone marrow stem cells. (A) Heat plot of lineage-specific genes from gene expression analysis of FACS-purified LSK/FLT3- cells pooled from 2 groups of 5 null mice were compared with LSK/FLT3- or LSK/FLT3+ cells pooled from $30 \mathrm{WT}$ mice. (B) Heat plot diagram of granulocyte/monocyte- and megakaryocyte-primed genes associated with the LMPP population.

erythroid progenitor cells when compared with the Vav:Hdac $3^{+/+}$ animals, yet some mice displayed an intermediate Ter $119^{+}$population (Supplemental Figure 1F).

Hdac 3 is required for hematopoietic cell fate decisions. To better define the hematopoietic defect in the Vav:Hdac3 ${ }^{-/-}$mice, we analyzed the early/stem progenitor cells using lineage and stem cell markers. To do this, bone marrow was first lineage depleted using the panel of lineage-positive antibodies (CD3, B220, MAC-1, GR-1, and Ter119) linked to magnetic beads, and the remaining cells were labeled using antibodies for SCA1 and c-KIT. The LIN-SCA1 ${ }^{+}$

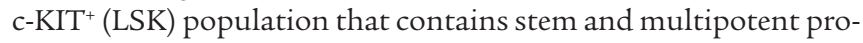
genitor cells showed a 2 -fold increase as a percentage of cells in the bone marrow (Figure 2A). Partitioning of the $\mathrm{LIN}^{-} / \mathrm{c}-\mathrm{KIT}^{+} / \mathrm{SCA} 1$ myeloid progenitor cells using $\mathrm{CD} 34$ and $\mathrm{F} \gamma \mathrm{R}$ expression detected a pronounced skewing of these progenitor populations, with more megakaryocyte-erythroid progenitors (MEPs) $\left(\mathrm{LIN}^{-} \mathrm{KIT}^{+} \mathrm{SCA}{ }^{-}\right.$ CD34- $\mathrm{F} \gamma \mathrm{R}^{\mathrm{lo}}$ ) and fewer granulocyte-macrophage progenitor cells (GMPs) (LIN-KIT $\left.{ }^{+} \mathrm{SCA} 1^{-} \mathrm{CD} 34^{+} \mathrm{Fc} \gamma \mathrm{R}^{\text {hi }}\right)$ and common myeloid progenitor cells (CMPs) ( $\mathrm{LIN}^{-} \mathrm{KIT}^{+} \mathrm{SCA} 1^{-} \mathrm{CD} 34^{+} \mathrm{Fc} \gamma \mathrm{R}^{\mathrm{med}}$ ) (Figure $2 \mathrm{~B})$. To better examine the lymphoid progenitor compartment, the LSK cells were subdivided using CD34 and FLT3. Remarkably, there was essentially a complete loss of LSK/FLT3 ${ }^{\text {hi }}$ cells in the bone marrow from mice lacking Hdac3 (Figure 2C). By contrast, there was little if any change in the $\mathrm{LSK} / \mathrm{CD} 34^{+}$population (Figure 2D). Similar results were obtained with an alternative strategy using anti-FLT3 coupled with the "slam" markers anti-CD150 and anti-CD48 $(43,44)$, which showed a loss of the $\mathrm{FLT3}^{+} / \mathrm{CD}^{+} 8^{+}$ CD150- population (Supplemental Figure 2).

In revised HSC fate maps, the LSK population was subdivided into LSK/FLT3 ${ }^{\text {lo }}$ MPP cells and LSK/FLT3 ${ }^{\text {hi }}$ lymphoid-primed MPP (LMPP) cells (39), then further subdivided using CD150 and CD48. Therefore, we used anti-CD48 (Slam48) and anti-CD150 (Slam150) to distinguish the progression of stem cells to MPP cells in Vav-Hdac3 $3^{-1-}$ mice $(43,44)$. This analysis indicated that the LSK/FLT3- population did not undergo the normal progression from LSK/FLT3-CD48-CD150+ (stem cells) to LSK/FLT3 $\mathrm{CD} 48^{+} \mathrm{CD} 150^{+}$(MPP1) to LSK/FLT3-CD48 ${ }^{+} \mathrm{CD} 150^{-}$(MPP2) and LSK/FLT3 ${ }^{+} \mathrm{CD} 48^{+} \mathrm{CD} 150^{-}$(LMPP), but accumulated at the MPP1 to MPP2 stage (Figure 2E and ref. 45). Quantification of actual cell numbers suggested that the $H d a c 3^{-/-}$bone marrow contained fewer stem cells (Figure 2E, graph), but given that the marrow is hypocellular with about half the normal complement of total cells, on a percentage basis, there were slightly more long-term HSCs in the null marrow. Based on these results, subsequent analyses used LSK/FLT3- cells, which encompass the stem cell populations and both the MPP1 and MPP2 cell populations.

Because of the dramatic loss of $\mathrm{B}$ and $\mathrm{T}$ cells that was linked to the loss of the LSK/FLT3 ${ }^{\text {hi }}$ population in the bone marrow, we crossed the $\mathrm{Hdac} 3^{f l / f l}$ mice with the interferon-inducible Mx1-Cre transgenic mice to confirm these results when $H$ dac 3 was deleted in adult mice. At 6 to 8 weeks after birth, poly pI:pC was injected 
A


B
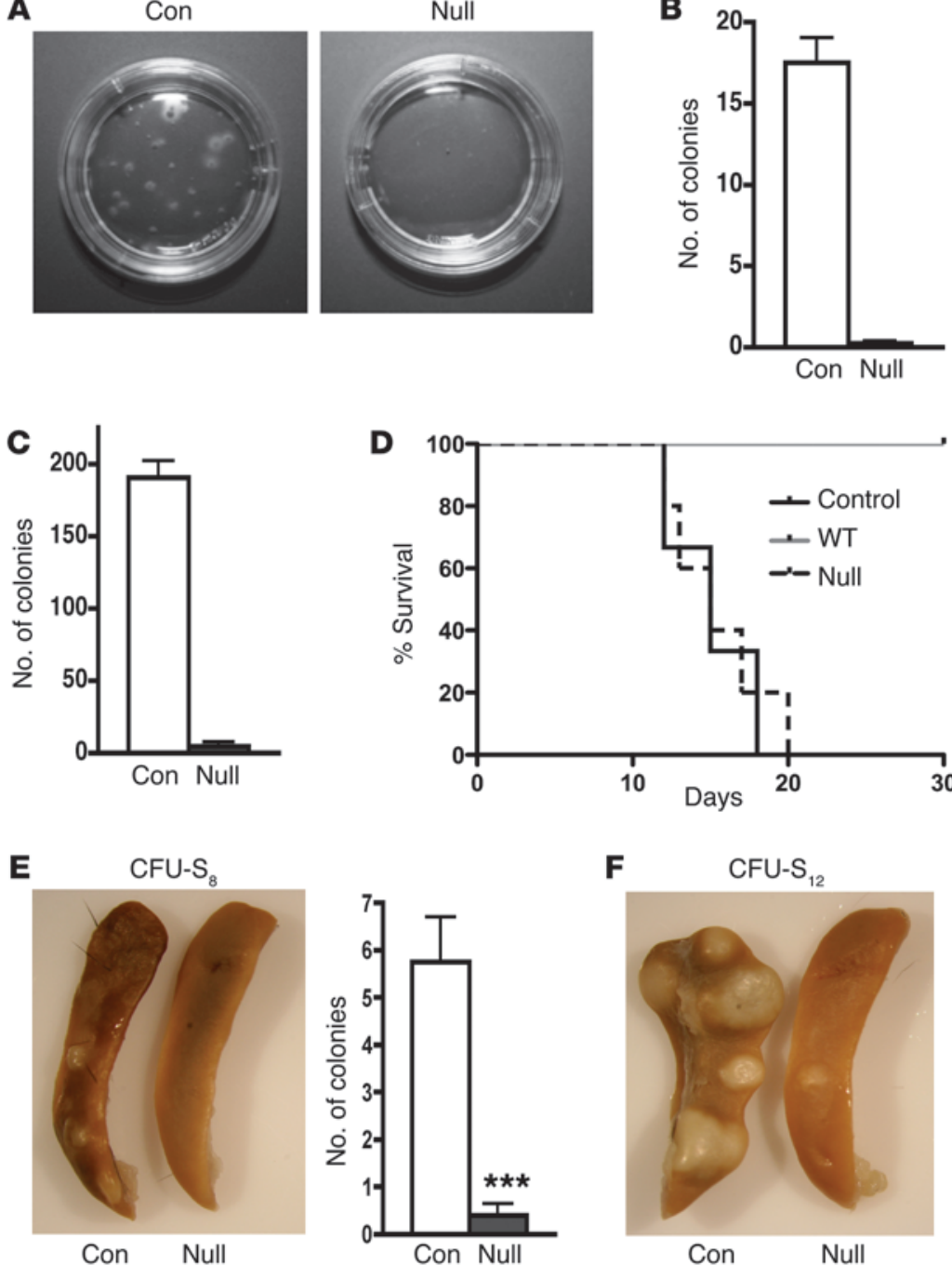
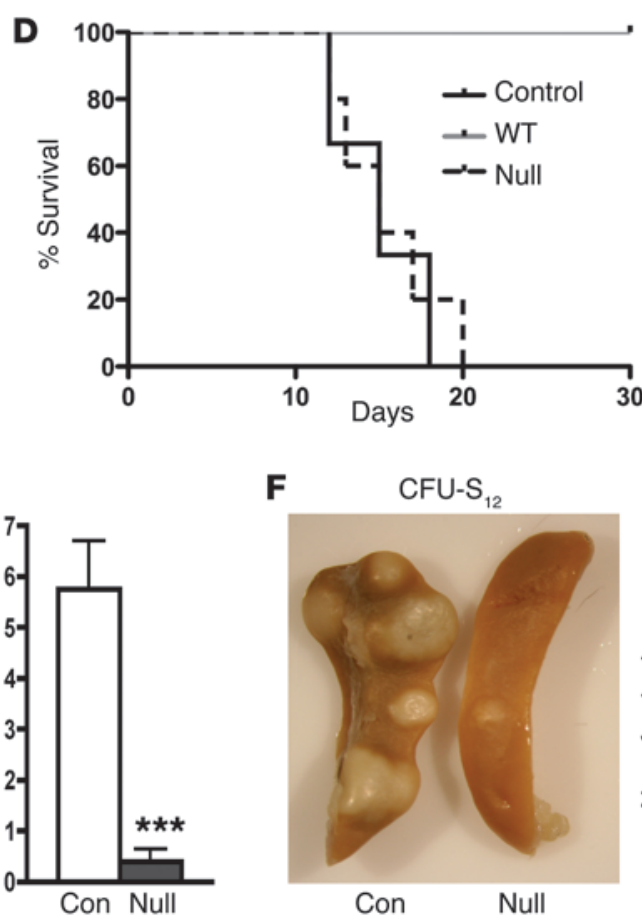

Figure 4

Loss of Hdac 3 disrupts hematopoietic progenitor and stem cell functions. (A) Myeloid progenitor cell colony formation assays. Bone marrow $\left(2.5 \times 10^{4}\right)$ cells were plated in methylcellulose containing IL-3, IL-6, Epo, and SCF, and colonies were quantified after 8 to 10 days in culture. Representative images of the plates are shown. (B) BFU-E was determined using methylcellulose containing Epo. (C) B cell progenitor colony formation assays. CFU pre-B cells that expanded in IL-7 were quantified after 7 days in culture. (D) Survival curves of a bone marrow transplant experiment using $1 \AA ̊ \sim 106$ WT (gray line) or Hdac3-null bone marrow cells (hatched line) and irradiation controls (black line). CFU-S were assessed on day $8(\mathbf{E})$ or day $12(\mathbf{F})$. Quantification of each CFU-S was calculated for the number of colonies per spleen and is shown as a bar graph (WT: $n=5$; Hdac3-null: $\left.n=5 ;{ }^{* \star \star} P=0.0005\right)$. Con, control. for 2 weeks, and the bone marrow was harvested and analyzed by flow cytometry. Once again, we used GFP expressed from the LoxStop-Lox-GFP allele to ensure that we assessed null cells (data not shown), and similar to the Vav-Cre mice, the marrow was hypocellular, with an increase in the undifferentiated $\mathrm{LIN}^{-}$population (Supplemental Figure 3, A and B). In addition, there was a significant increase in the LSK population and a loss of $\mathrm{Hdac}^{-/-} \mathrm{B} 22 \mathrm{O}^{+}$ cells in the marrow (Supplemental Figure 3, C and D). Furthermore, when we transplanted $\mathrm{M} x 1-\mathrm{Cre}: \mathrm{Hdac} 3^{\mathrm{Fl} / F l}$ bone marrow into recipient mice prior to induction of cleavage, we obtained the same phenotype, including a block in the formation of LSK $/ \mathrm{FLT}^{+}$cells (Supplemental Figure 4). Thus, the defect is intrinsic to hematopoietic stem and progenitor cells, and the timing of removal of Hdac3 did not affect the phenotype, but the Vav-Cre allele did yield a more penetrant phenotype.

The Mx1-Cre/Hadc3 $3^{-/}$mice also allowed us to examine the fate of the LMPPs and early B cell progenitor cells that direct B cell development immediately following deletion. FACS analysis performed 6 days following the last injection of poly pI-pC to induce $\mathrm{Hdac} 3$ deletion showed a similar percentage of mature $\left(\operatorname{IgM} \mathrm{Mi}^{\mathrm{hi}} \mathrm{B} 20^{\mathrm{hi}}\right)$ cells, but reductions in the immature populations (Supplemental Figure 5, A and B). In addition, there was no evidence of increased apoptosis within the bone marrow B220 $\mathrm{B}$ cell populations and no changes in the numbers of $\mathrm{CD}^{+}$splenic T cells or B220+ splenic B cells (Supplemental Figure 5C). Taken together, these data suggest that the LMPP and immature $\mathrm{B}$ cells present during the induction of $\mathrm{Hdac} 3$ deletion continued to mature rather than undergo rapid apoptosis, and the mature cells were not affected, which is consistent with a block in the formation of LSK/FLT3 ${ }^{+}$(LMPP) cells.

Inactivation of Hdac3 alters gene expression in LSK/FLT3- cells. Nuclear hormone receptors recruit $\mathrm{Hdac} 3$ to regulate myelopoiesis (e.g., the retinoic acid receptor), suggesting that inactivation of Hdac3 might stimulate a myeloid gene expression program at the expense of lymphopoiesis. Therefore, we compared gene expression in LSK/FLT3- cells from null mice with LSK/FLT3 ${ }^{-}$and LSK/ $\mathrm{FLT}^{+}$control cells to molecularly characterize the defects in the Vav:Hdac3 $3^{-/}$MPP populations. Cells were FACS purified from 10 null mice ( 2 sets of 5 mice) and 30 control mice. These purified populations were pooled before RNA was prepared and hybridized to Affymetrix microarrays. Spearman's correlation analysis indicated that there were not dramatic changes in gene expression (Spearman's correlation coefficient of 0.98) (Supplemental Figure 6). However, analysis of previously identified genes that are 
A

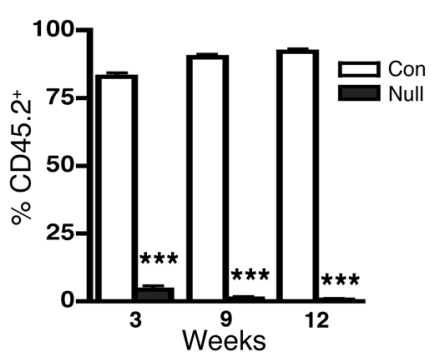

C

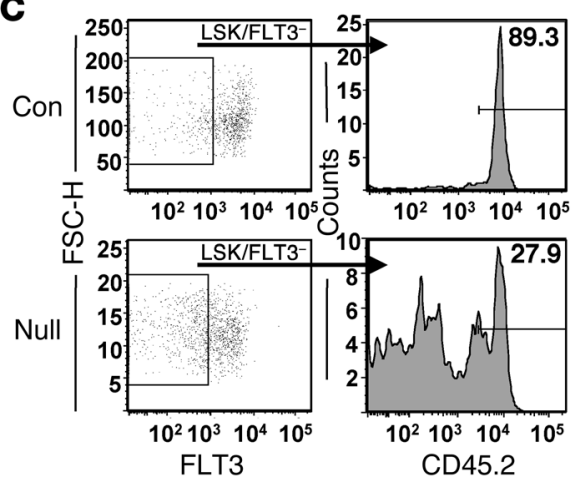

B

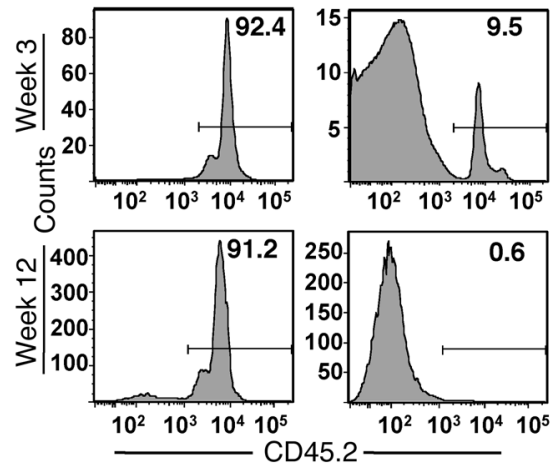

D

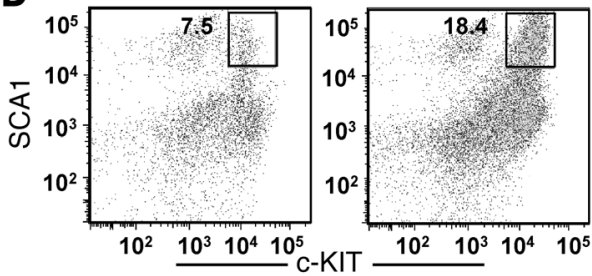

\section{Figure 5}

Hdac3 is required for stem cell functions. (A) Competitive repopulation assay in which $90 \%$ control (empty bars) or $\mathrm{Hdac3}^{-/-}$(filled bars) CD45.2+ bone marrow cells were coinjected with $10 \%$ WT CD45.1+ cells, and peripheral blood was analyzed by FACS. Data shown are the mean \pm SEM at times after transplantation $\left({ }^{* * *} P \leq 0.001 ; n=4\right)$. (B) FACS analysis of bone marrow to determine the percentage of cells that were CD45.2+ after a competitive repopulation assay. (C) FACS analysis of LSK/FLT3- cells to determine the percentage of CD45.2+ cells that had repopulated the marrow 3 weeks after cBMT. (D) FACS analysis of bone marrow of control and Mx1-Cre:Hdac3-1- mice for $\mathrm{GFP}^{+}$cells (for $\mathrm{D}: \mathrm{WT}=60 \% \mathrm{GFP}^{+}$, null $=78 \% \mathrm{GFP}^{+}$) that were $\mathrm{LIN}^{-}$and SCA1 ${ }^{+}$ and $\mathrm{C}-\mathrm{KIT}^{+}$. (E) Graphs of the numbers of colonies formed in methylcellulose at the indicated times from LTC-IC cultures of WT (open bars) and $\mathrm{Hdac}^{-/-}$(filled bars) bone marrow cells, or WT cells treated with the indicated HDAC inhibitors $\left({ }^{* * *} P=0.001, n=3 ;{ }^{*} P=0.013\right.$, week 3 , $n=3 ;{ }^{\star *} P=0.006$, week $2 ; P=0.002$, week $3 ; P=0.019$, week $4, n=3$ ). Representative results from 2 separate experiments are shown. The numbers in each box are the relative percentage of the cells in the indicated gated population. Untr, untreated.
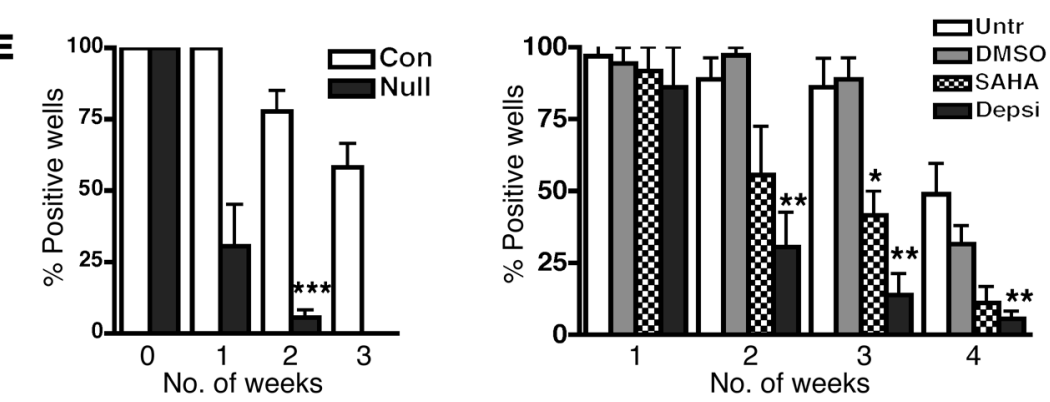

characteristically expressed in stem cells, myeloerythroid, and lymphoid progenitor cells (46-48) showed some upregulation of genes characteristic of stem cells (e.g., Runx1, Wnt3a, Wnt1; Figure 3 and Supplemental Table 1). Myeloid primed genes such as Cebpa and Cebpe were upregulated along with $C \mathrm{cr} 2$, but others such as $C s f 1 r$ were unchanged or slightly lower in $\mathrm{Hdac3}^{-/-}$LSK/FLT3- cells (Figure 3 and Supplemental Tables 1 and 2). Some MEP genes were downregulated in the absence of Hdac3 (Figure 3, Supplemental Tables 1 and 2, and ref. 49), which is consistent with the anemia observed in the null mice. Quantitative RT-PCR (qRT-PCR) was used to confirm the microarray results using RNA isolated from a separate cohort of $30 \mathrm{WT}$ and 2 null mice (e.g., Dntt reduced 38 fold; Ccr 2 increased 4 fold; Slamf1 [CD150] increased 2.5 fold).

Most notably, lymphoid-specific genes were downregulated in $\mathrm{Hdac}^{-/-}$cells, as compared with WT LSK $/ \mathrm{FLT}^{+}$cells, which provides a molecular indicator for the loss of the LMPP cells (Figure 3B, compare lanes labeled "Null" with lane labeled "FLT3+"). For example, Dntt, Rag2, Satb1, and Flt3 were downregulated in Hdac3-null LSK/FLT3- cells as compared with the control LSK/FLT3- cells, but were even lower when compared with the WT LSK/FLT3 ${ }^{+}$cells. The lymphoid "primed" genes (Cnn3, Crip1, Emp1, Irf8, Blnk, and Ets1) were also lower in Hdac3-/LSK/FLT3- cells (Figure 3A). Thus, the $\mathrm{Hdac}^{-/-}$stem cells did not simply fail to express markers of lymphoid development such as Flt3, but failed to initiate the "priming" necessary for these cells to mature into LMPP cells.

$H d a c 3$ is required for the proliferation of HSCs and progenitor cells. Next, we assessed hematopoietic progenitor cells from the Vav-Hdac3-1 mice using methylcellulose colony formation assays. When methylcellulose containing IL-6, SCF, GM-CSF, and Epo was used to culture myeloid progenitor cells, the number of colonies that formed from $\mathrm{Vav}-\mathrm{Hdac} 3^{-/-}$bone marrow was similar to that derived from control bone marrow, but the colonies were distinctly smaller, suggesting a general proliferation defect (Figure 4A). The small colony size made phenotyping difficult, but it appeared that the most differentiated CFU monocyte (CFU-M) and CFU granulocyte (CFU-G) populations were present. However, there was an absence of colonies that contained erythroid cells, including erythroid burst-forming units (BFU-Es) and CFU granulocytes, erythrocytes, monocytes, megakaryocytes (CFU-GEMMs, or a multipotential progenitor cell colony) (Figure 4A and data not shown). Therefore, we used methylcellulose containing only Epo to assess erythropoietic progenitor growth, which yielded no BFU-Es (Figure 4B). Additionally, we examined B cell formation using methylcellulose containing IL-7 and confirmed that $H d a c 3$ was required for the formation of B cells, as very few colonies were present (Figure 4C). 
A

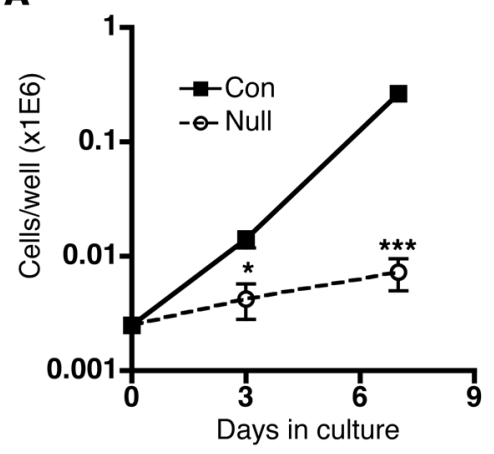

B
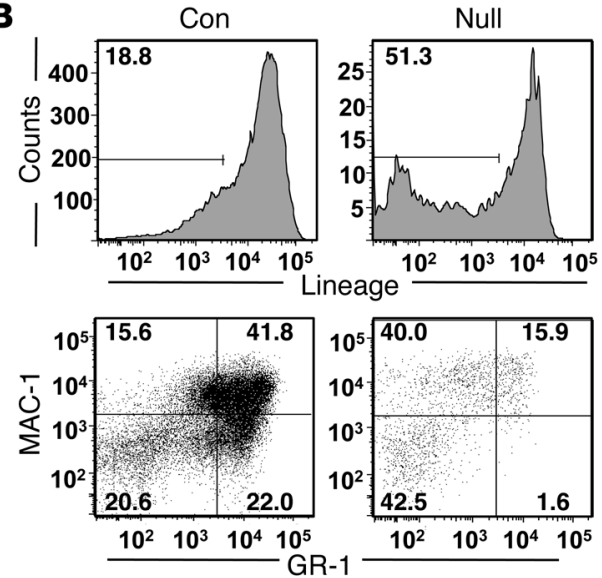

Day 7 cultures
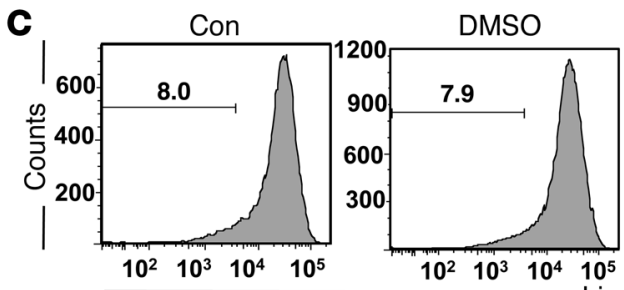

DMSO
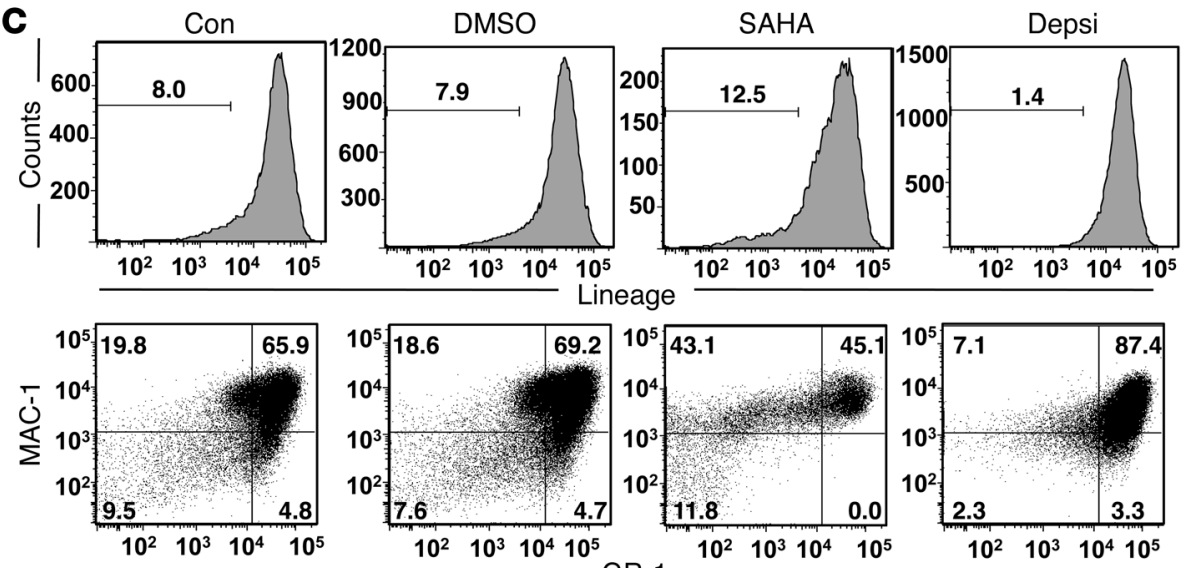

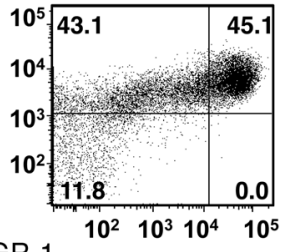

GR-1- 7 -

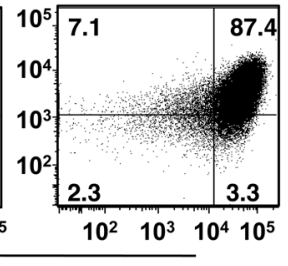

Day 7 cultures

\section{Figure 6}

Inactivation of Hdac3 impairs progenitor cell proliferation differentiation. (A) 2,500 WT or Hdac3-null LSK/FLT3- bone marrow cells were cultured on an OP9GFP stromal layer in media containing IL-6, SCF, and LIF, and proliferation was monitored by counting the total number of cells 3-7 days after harvesting the bone marrow cells (day $3, P=0.026$; day $7, P \leq 0.001 ; n=3$ ). (B) Flow cytometric analysis of day-7 cultures (from $\mathbf{A}$ ) using a combination of anti-CD3, anti-B220, anti-GR-1, anti-MAC-1, and anti-Ter119 to distinguish mature cells from immature progenitor cells (top panel) or a combination of anti-GR-1 and anti-MAC-1 to quantify myeloid cell differentiation (bottom panel). (C) Flow cytometric analysis of WT LSK cells treated with DMSO, SAHA, or depsipeptide (Depsi) and cultured for 7 days as in (A). Representative plots show a combination of anti-CD3, anti-B220, anti-GR-1, anti-MAC-1, and anti-Ter119 to distinguish mature cells from immature progenitor cells (top panel) or a combination of anti-GR-1 and anti-MAC-1 to quantify myeloid cell differentiation (bottom panel). The numbers in each box indicate the relative percentage of the cells in the indicated gated population.
Given that inactivation of Hdac 3 caused genomic instability and apoptosis in murine embryonic fibroblasts (MEFs) (31) and that hematopoietic progenitor cells displayed a growth defect, we functionally tested the short-term stem and progenitor cell populations. Bone marrow transplantation (BMT) assays were used to assess the ability of stem and progenitor cells to repopulate lethally irradiated mice. Remarkably, irradiated mice injected with a million Hdac3-null bone marrow cells had survival curves that matched those of the lethally irradiated control mice, whereas mice injected with control bone marrow cells were rescued by the transplant (Figure 4D). Therefore, we performed spleen colonyforming assays to quantitatively measure the response to hematopoietic stress after BMT. The Hdac3-null bone marrow yielded essentially no colonies at either 8 days after transplantation, which measures MEP cells (Figure 4E), or at 12 days after injection when the colonies formed represent both MEPs, MPP cells, and shortterm stem cells (Figure 4F and ref. 50).

$H$ dac 3 is required for long-term stem cell functions. Given the dramatic growth defects of MPP cells, we used competitive BMT (cBMT) assays to provide the null stem cells with WT progenitor cells in order to measure long-term stem cell functions. We injected a mixture of $90 \%$ Vav:Hdac3 $3^{-/}$bone marrow cells that express CD45.2 and $10 \%$ WT CD $45.1^{+}$cells into lethally irradiated mice. Only 3 weeks after transplantation into the CD $45.1^{+}$recipient mice, we observed a prominent decline in the numbers of CD45.2+ $\mathrm{Hdac3}$ null bone marrow cells in the peripheral blood, which continued to worsen with time (Figure 5A). FACS analysis of the bone marrow 3 weeks after transplantation showed that only about $10 \%$ of the cells were generated from Hdac3-null bone marrow cells (Figure $5 \mathrm{~B})$. By 12 weeks, when most cells are derived from the transplanted stem cells, essentially all of the $\mathrm{Hdac3}^{-/-}$cells were outcompeted by the 10\% WT marrow (Figure 5B). Moreover, within the LSK/FLT3- ${ }^{-}$population, by 3 weeks after transplantation, the percentage of $\mathrm{Hdac3}^{-/-}$cells had dropped to $25 \%$ to $30 \%$ (Figure 5C). Given that $\mathrm{Hdac}^{-/-}$bone marrow contains roughly 3-4 times the number of LSK/FLT3- cells, this population declined at a rate similar to that of $\mathrm{Hdac}^{-/-}$cells in the peripheral blood, which are primarily lymphocytes (Figure 5A), suggesting that the MPP1/2 populations were defective in their ability to proliferate.

The competitive stem cell transplant also indicated that Hdac 3 is required for the function of stem cells and that this defect is intrinsic to the stem cell. However, the defect was so severe that we were unable to assess the function of other progenitor cells downstream of the stem cells after transplantation. Therefore, we returned to the Mx1-Cre:Hdac3 ${ }^{-/}$model and used the Stop-Lox-Stop-GFP allele to monitor Cre action and identify mice that contained only $60 \%-$ $80 \% \mathrm{GFP}^{+}$cells, yielding chimeric marrow. Even in the presence of some WT progenitor cells (e.g., for the analysis in Figure 5D: 

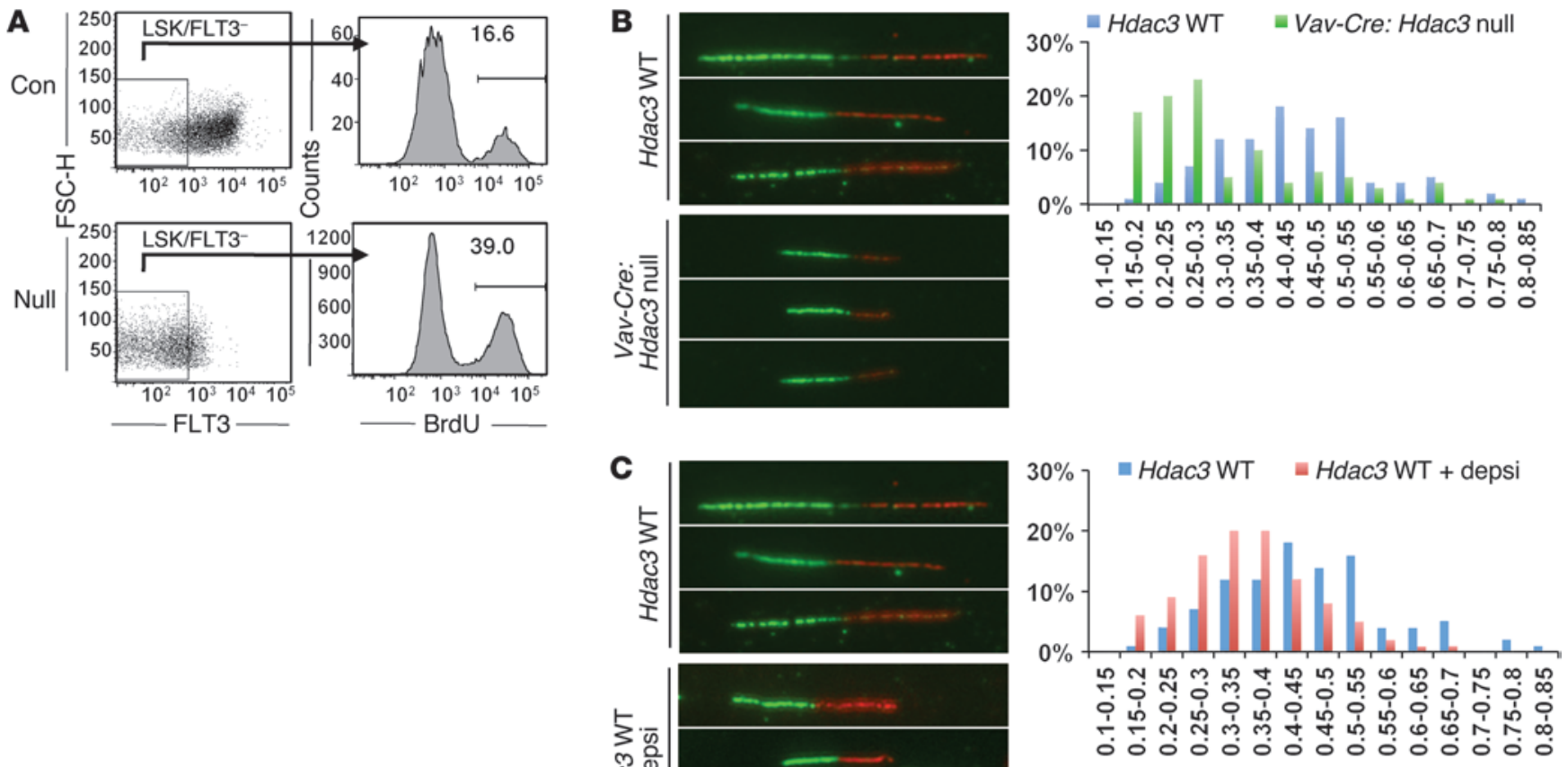

\section{Figure 7}

Hdac3-null stem cells are cycling and show defects in DNA replication. (A) Cell cycle status of LSK/FLT3- cells was analyzed using BrdU. A representative FACS plot is shown from an experiment performed with 4 mice that is consistent with other biological replicates. The numbers in each box are the relative percentage of the cells in the indicated gated population. (B) DNA fiber labeling with IdU (green) and CldU (red) was used to assess DNA replication fork progression in LSK/FLT3- cells from control and $\mathrm{Hdac}^{-/-}$bone marrow. Graphical representation of the length of replication tracks taken from 100 DNA fibers is shown in the graph. (C) DNA fiber labeling of LSK/FLT3- cells as in B, but using untreated controls or cells treated with depsipeptide for 5 minutes prior to the addition of IdU with continued treatment with depsipeptide throughout the labeling period. A nonparametric Mann-Whitney $U$ test indicated that the differences were significant at the $P=0.001$ level for $\mathbf{B}$ and $\mathbf{C}$.

$78 \% \mathrm{GFP}^{+}, 22 \% \mathrm{GFP}^{-}$for the null sample), the $\mathrm{Hdac3}^{-/-}$LSK cells expanded 2-to-3 fold (Figure 5D), suggesting that this was not due to a problem that had accumulated during development or that can be complemented by WT cells. Similar results were obtained after transplantation of $\mathrm{M} x 1$-Cre:Hdac $3^{f l / f l}$ bone marrow prior to deletion (Supplemental Figure 4A), which recapitulated the VavCre phenotype of a block at the LSK/FLT3- ${ }^{-}$stage and further indicated a cell-intrinsic defect. When bone marrow progenitor cells were labeled with the vital dye CFSE prior to transplantation, a similar number of null cells homed to the marrow as in the WT controls (data not shown), indicating that the stem cell defect was unlikely to be due to a homing defect.

To extend these results, we used in vitro long-term culture of initiating cell (LTC-IC) assays to measure stem cell activity outside of microenvironmental influences. Cultures of cells lacking Hdac3 rapidly lost self-renewal capacity, whereas control cells retained multilineage potential for multiple weeks (Figure 5E). This defect in $\mathrm{Hdac}^{-/-}$stem cell function was similar to the effect of a 48-hour pretreatment of control cultures with the HDAC inhibitors SAHA (vorinostat) or depsipeptide (romidepsin) (Figure 5E, right panel).

Although we noted changes in differentiation and gene expression, the proliferation defects appeared to be the most likely mechanism underlying the stem cell defect observed in cBMTs. Therefore, we cultured 2,500 LSK/FLT3- cells from control or Vav:Hdac $3^{-/}$mice on OP9 stromal cells with media containing
IL6, SCF, and LIF to more carefully examine cell cycle progression versus myeloid differentiation and to further define the effect of Hdac3 inactivation in MPP cell functions. Whereas control stem/ progenitor cells underwent a rapid expansion to over 150,000 cells within 1 week when provided with signaling to cycle, there was a profound defect in the expansion of this population from $\mathrm{Hdac}^{-/-}$ mice in culture (Figure 6A).

This lack of proliferation was consistent with growth defects observed in spleen colony-forming units (CFU-S) and methylcellulose colony formation (Figure 4), and cell cycle defects due to $\mathrm{S}$ phase-associated DNA damage observed in $\mathrm{Hdac3}^{-/-}$fibroblasts (31). Intriguingly, the majority of the null cells remained negative for lineage markers (Figure 6B, upper panels), suggesting that a major defect in differentiation led to the accumulation of LSK/FLT3- cells in vivo, rather than hyperproliferation to compensate for downstream defects. This result is consistent with the observation of an expansion of LSK cells in Mx1-Hdac $3^{-/-}$mice, even when the percentage of cleavage was lower, leaving WT progenitor cells to complete lineage allocation (Figure 5D). However, the cells that did differentiate in these cultures were primarily GR-1/MAC-1 double positive or MAC-1-positive (Figure 6B, lower panels), which was also observed in vivo (Figure 1D). This is similar to the effects observed when WT LSK cells were treated with SAHA, which caused a slight increase in the number of immature, $\mathrm{LIN}^{-}$cells (Figure 6C, upper panels) and skewing toward $\mathrm{MAC}-1^{+}$and $\mathrm{GR}-1^{+} / \mathrm{MAC}-1^{+}$cells (Figure $6 \mathrm{C}$, lower 
panels). Intriguingly, depsipeptide $(23,24)$ impaired proliferation but did not cause the accumulation of immature cells or the same type of skewing toward MAC-1 $1^{+}$cells, but increased the number of GR $-1^{+} / \mathrm{MAC}^{-1^{+}}$double-positive cells (Figure 6C). This effect was dose dependent (Supplemental Figure 7) and suggests that even low amounts of this potent $H d a c$ inhibitor trigger differentiation.

Inactivation of Hdac3 impairs S phase progression of MPP cells. Inactivation of Hdac 3 caused an accumulation of LSK/FLT3- progenitor cells (Figure 2), yet these cells showed dramatic defects in reconstituting hematopoiesis after BMT and defects in proliferation (Figures 4 and 5). To explore the mechanism behind these defects, we injected Vav:Hdac3 ${ }^{-/-}$and control mice with BrdU to assess the number of LSK/FLT3- stem cells that were in the S phase. Remarkably, only 2 hours after injection of BrdU, 39\% of the null stem/ progenitor cells incorporated BrdU into their DNA (Figure 7A), suggesting that this population was actively cycling. Similar results were obtained using CD150 versus CD48 expression (Supplemental Figure $8 \mathrm{~A}$ ), which suggests that both stem and progenitor cells from Vav:Hdac3 $3^{-/-}$had nearly twice the number of cells in the $\mathrm{S}$ phase. Although less dramatic, a similar trend was also observed in the $M x 1$-Cre-inducible system, with the largest increase observed in the MPP2 population (Supplemental Figure 8B).

While there were large numbers of $\mathrm{Hdac}^{-/-}$stem cells in the $\mathrm{S}$ phase, the failure of these cells to proliferate in vitro (Figure $6 \mathrm{~A})$ suggests that these cells were not completing the cell cycle in a timely fashion. Therefore, we tested whether this population of cells was capable of robust DNA replication using DNA fiber labeling, which uses thymidine analogs that can be detected by antibodies to examine the progression of individual replication forks. Bone marrow cells were cultured in vitro for 20 minutes in the presence of the thymidine analog IdU, followed by a second 20-minute labeling with CldU. LSK/FLT3- cells were then isolated, and at least 100 individual DNA fibers were analyzed with anti-IdU (green) and anti-CldU (red) to assess DNA replication fork progression (Figure 7B). While WT cells robustly incorporated these nucleotide analogs, DNA from $H \mathrm{dac}^{-/-}$mice displayed dramatically shorter strand labels. These data indicate that Hdac3 is required for efficient DNA replication in the stem/progenitor cell pool, which likely accounts for the impaired proliferation of these cells.

Finally, we tested whether HDACs act at the level of chromatin structure or are required at the replication fork for efficient replication fork progression. We reasoned that global changes in chromatin structure would require at least a few hours to occur, which is the time frame required for histone acetylation to accumulate in the cell, whereas activity needed at the replication fork would be immediate for cells in the S phase. WT LSK/FLT3- cells were isolated from the bone marrow and treated with depsipeptide for only 5 minutes prior to the addition of IdU followed by labeling with CldU. DNA fiber analysis indicated that even a brief pretreatment with depsipeptide affected replication fork progression to an extent similar to that of Hdac3 deletion (Figure 7C). These data imply that HDAC activity is needed at or near the replication fork to maintain efficient DNA replication in stem/MPP cells.

\section{Discussion}

In order for stem cells to repopulate the bone marrow, they must yield progeny that can undergo massive proliferation and at the same time self-renew to maintain the stem cell pool. Deletion of Hdac3 in the HSC caused anemia and hypocellular marrow. In BMT assays, CFU-S assays, and in vitro, Hdac 3 was essential for the proliferation of stem and MPP cells and the production of LMPP cells, as well as for mature erythroid cells that are needed for animals to thrive. In humans, similar bone marrow failure syndromes are commonly due to defective genomic stability, and HDAC3 lies within a region on Ch. $5 \mathrm{q} 31$ that is commonly deleted in myelodysplastic syndromes (51), suggesting that loss of HDAC3 expression could contribute to these phenotypes.

Mechanistically, we found that over one-third of $\mathrm{Hdac}^{-/-}$stem/ progenitor cells were labeled by a pulse of BrdU in vivo, yet failed to proliferate in vitro, which is consistent with the idea that these cells were impaired in their progression through the S phase. Using DNA fiber-labeling assays, we were able to show that these stem/ progenitor cells were unable to efficiently replicate their DNA in vitro. Moreover, depsipeptide, which targets all class 1 HDACs, also affected replication fork velocity, even when added just before the thymidine analog. These data suggest an unexpected requirement for Hdac3 during DNA replication. Although Hdac3 has been found at the replication fork (52), it did not appear to move with the fork. Taken together with our data, it is possible that Hdac3 modifies chromatin both in front of and behind the fork to ensure the unimpeded movement of the fork and correct chromatin formation of the newly replicated DNA. Nevertheless, these data suggest that DNA replication is a primary and immediate target of depsipeptide and other HDAC inhibitors being developed for cancer therapy.

Given the expectation that loss of Hdac3 would stimulate retinoic acid receptor-mediated transcriptional programs that are associated with myeloid differentiation, it is surprising that many Hdac3-deficient LSK/FLT3- cells failed to commit to a specific lineage and remained undifferentiated in vitro (Figure 6), accumulated at the MPP1/2 stage in vivo (Figure 2, 5), and maintained a gene expression signature consistent with the earliest MPP cells or stem cells (Figure 3). Importantly, competitive bone marrow reconstitution assays demonstrated a dramatic loss of Hdac3-null stem cell functions in the presence of WT progenitor cells. The combination of these data suggests that $H d a c 3$ is required for cell-intrinsic stem cell functions, which are consistent with defects in DNA replication (Figure 7).

Vav:Hdac3 $3^{-1-}$ mice lacked a functional thymus and contained almost no circulating $\mathrm{T}$ cells, B cells, or lymphoid progenitor cells (Figure 1). This result is consistent with a loss of the LSK/FLT3 ${ }^{+}$ "LMPP cell", which has been proposed as a B and T progenitor cell that also contributes to the GMP population (39). Indeed, we also noted reduced cellular allocation to the GMP population (Figure 1). Additionally, gene expression data showed that genes associated with lymphoid "priming" were downregulated in the Hdac3-null LSK/FLT3- cells (Figure 3 and ref. 46). Taken together, the FACS and microarray data show that loss of Hdac3 specifically caused the loss of $\mathrm{B}$ and T cells by disrupting the formation of LSK/ FLT3+"LMPP" cells. This conclusion is supported by the inducible deletion of Hdac3 in the Mx1-Cre model (Supplemental Figure 5), and further T and B cell-specific deletion of Hdac3, which did not trigger cell death, but impaired the development of thymic T cells (Summers, unpublished observations) or the function of splenic B cells (Bhaskara and Stengel, unpublished observations).

These results are also consistent with the "myeloid-based" model of hematopoiesis (53). That is, one possible explanation for the loss of lymphoid potential is that inactivation of Hdac 3 triggers severe proliferative stress on the hematopoietic system, such that those cells that pass the differentiation block in the stem cell compartment are routed into the erythroid lineage to allow the mice 
to survive. However, the findings from the gene expression studies and in vitro differentiation suggest that inactivation of Hdac3 may also create a transcriptional program (e.g., upregulation of Cebpa and Cebpe) (Figure 3 and Supplemental Table 1) that drives cells to become myeloid, rather than contribute to lymphopoiesis. Importantly, these defects do not simply reflect the failure to express Flt3, as stem cells lacking this tyrosine kinase receptor were able to produce lymphoid progeny (54).

The dramatic failure of transplanted $\mathrm{Hdac}^{-/-}$bone marrow cells to expand prevented a full analysis of the ability of these null stem cells to undergo self-renewal. However, the LTC-IC assays are one measure of this functionality, and the loss of Hdac3 dramatically impaired the ability of cells to maintain pluripotency, even more so than treatment with SAHA or depsipeptide (Figure 5E). The use of these HDAC inhibitors is associated with adverse hematopoietic side effects that are similar to the phenotypes observed in $\mathrm{Hdac3}^{-/-}$ mice. Ten percent to $20 \%$ of the patients experienced thrombocytopenia and anemia, with some patients experiencing lymphopenia (55-57). Our results indicate that inhibition of Hdac 3 is one of the causes of these phenotypes, since the Vav-Hdac3 $3^{-1-}$ mice showed signs of anemia, and there were dramatic defects in MEP cell expansion in CFU-S assays and in methylcellulose colony-formation assays (Figure 4). Although lymphopenia is only a problem for a portion of patients taking these drugs, the profound defect in the production of $\mathrm{B}$ and $\mathrm{T}$ cells in the knockout mice indicates that inhibition of $\mathrm{Hdac} 3$ also contributes to this side effect.

The ability to remodel chromatin is a key feature that stem cells must maintain to ensure a pluripotent state and the ability to self-renew while yielding progeny that can go on to produce all of the mature hematopoietic lineages. Our data hint at how inhibiting Hdac3 may be beneficial for the treatment of hematopoietic malignancies. By affecting stem cell self-renewal and DNA replication, inhibition of $\mathrm{Hdac} 3$ might represent a rational approach for treating myeloid leukemia, especially those that show characteristics of immature myeloid progenitor cells (e.g., M0, M1, and M2). In fact, the immediate effect of these inhibitors appears to be on DNA replication rather than on changes in gene expression that may rely on changes in histone acetylation (Figure 7C). Thus, $\mathrm{Hdac} 3$-selective inhibitors may be useful in myeloid leukemia as both antiproliferative agents and as differentiation agents.

\section{Methods}

Mice. Mice harboring a conditional allele $\left({ }^{\mathrm{fl}}\right)$ or a null allele $\left(^{-}\right)$of Hdac3 were crossed to ROSA26-Lox-Stop-Lox-GFP transgenic mice. The offspring from these mice were crossed to transgenic mice expressing Vav-Cre (38). Mice with a conditional allele in conjunction with a WT $\left({ }^{\mathrm{fl} /+}\right)$ or null allele $\left({ }^{(1 /-)}\right), G F P^{+/+}$, and Cre were obtained. Hdac $3^{f / f l}: G F P^{+/+}$mice were also crossed with mice expressing Mx1-Cre (58). To induce Cre expression in Mx1-Creexpressing mice, 6- to 8-week-old animals received an i.p. injection of $500 \mu \mathrm{g}$ poly pI:pC in PBS every other day for 13 days, for a total of 7 injections, with the last day of injection being denoted as day 0 . Mice were analyzed 2-4 days after the last injection.

Flow cytometric analysis. Single-cell suspensions were formed after the tibia and femur were flushed with PBS to collect bone marrow cells or after the spleen or thymus was minced into fragments and passed through a $70-\mu \mathrm{m}$ filter. Red blood cells were lysed with erythrocyte lysis buffer (buffer EL; QIAGEN). For lineage labeling, cells were stained with biotinylated antibodies against lineage-specific cell surface markers (CD3, B220, GR-1, MAC-1, and Ter119; eBioscience), followed by fluorochrome-conjugated streptavidin. For all other flow cytometric experiments, cells were labeled with the appropriate combination of fluorochrome-conjugated anti-c-KIT, anti-SCA1, anti-FLT3, anti-CD34, anti-FcyR, anti-CD150, anti-CD48, anti-CD4, anti-CD8, anti-GR-1, antiMAC-1, anti-B220, anti-CD3, anti-Terr119, and anti-CD45.2 (eBioscience). Analysis was performed with a 3-laser BD LSR II using FACSDiva and FlowJo software. For rare populations of cells, bone marrow samples were first lineage depleted using a lineage depletion kit and magnetic cell sorting (Miltenyi Biotec). Sorting of hematopoietic cell populations was performed on a BD FACSAria. For the BrdU incorporation assays, mice were sacrificed 2 hours after i.p. injection of $1 \mathrm{mg}$ of BrdU. Cells were then harvested as previously described and labeled with anti-BrdU (Flow Kit; BD Biosciences - Pharmingen).

Microarray and real-time $q P C R$. Bone marrow cells were harvested, and the $\mathrm{LIN}^{-}$fraction was separated using the Lineage Cell Depletion Kit and MACS columns (Miltenyi Biotec). The LIN- fraction was then stained with antibodies for flow cytometry and the LSK/FLT3 cells were sorted on a BD FACSAria. Total RNA was isolated from the sorted bone marrow cells using a PerfectPure RNA extraction kit (5 Prime). LSK/FLT3- cells pooled from 2 groups of 5 null mice were compared with LSK/FLT3- ${ }^{-}$or LSK/FLT3 ${ }^{+}$cells pooled from $30 \mathrm{WT}$ mice. The expression of individual genes was verified using RT-PCR. The microarray data were deposited in the Gene Expression Omnibus database (GEO accession number GSE45952).

Stem cell and progenitor cell assays. For the progenitor colony-forming cell assays, single-cell suspensions were obtained by flushing cells from the tibia and femur of mice. The red blood cells were lysed with Erythrocyte Lysis Buffer (Sigma-Aldrich). The cells were mixed with methylcellulose media containing rmSCF, rmIL-3, rhIL-6, rhEpo (Methocult GF M3434; Stem Cell Technologies), a CFU pre-B-specific methylcellulose containing rhIL-7 (Methocult GF M3630; Stem Cell Technologies), or a BFU-E-specific methylcellulose (Methocult SF M3436; Stem Cell Technologies).

For BMT, bone marrow cells were injected via the tail vein into lethally irradiated ( 9 Gy) recipient WT C57Bl/6 mice. For the evaluation of CFU-S abilities, $5 \times 10^{4}$ bone marrow cells derived from either control or $\mathrm{Hdac} 3$ null mice were transplanted, and the spleens were isolated 8 or 12 days after transplantation and fixed in Tellsniczky's fixative. For competitive reconstitution assays, lethally irradiated $\mathrm{C} 57 \mathrm{Bl} / 6 \mathrm{CD} 45.1^{+}$congenic mice were used as recipients. The control or Hdac3-null donor cells were mixed with C57Bl/6 CD45.1 bone marrow cells (9:1). Reconstitution by the donor (CD45.2) cells was monitored by flow cytometry of the peripheral blood and bone marrow.

For the LTC-IC assay, $3 \times 10^{3} \mathrm{LIN}^{-}$cells were cultured on OP9 stromal cells and weekly semireplenishment of media was performed using the StemCell Technologies procedure. At 1-week intervals, a set of wells was harvested, and the cells were plated in methylcellulose media (Methocult GF M3434; StemCell Technologies) and cultured for 10 days to determine the percentage of positive wells. For the Hdac inhibitor treatment in the LTC-IC assay, control LIN ${ }^{-}$cells were cultured on OP9 stromal cells in RPMI media supplemented with $10 \%$ FBS, $1 \%$ Pen-Strep, $1 \%$ L-Glut, $5 \mathrm{ng} / \mathrm{ml}$ IL-6, $50 \mathrm{ng} / \mathrm{ml} \mathrm{SCF}$, and $10^{3} \mathrm{U} / \mathrm{ml} \mathrm{LIF}$ for 48 hours in the presence of the drugs (SAHA, $1 \mu \mathrm{M}$; depsipeptide, $10 \mathrm{nM}$ ). After the 48-hour treatment, the cells were collected and counted for plating in the LTC-IC assay as described above.

For the in vitro culture of HSCs, OP9 stromal cells were grown to confluency and irradiated (20 Gy). After irradiation of the stromal cells, bone marrow cells were harvested and sorted as described above, and 2,500 LSK/ FLT3- cells were plated on top of the OP9 cells in RPMI media supplemented with $10 \%$ FBS, $1 \%$ Pen-Strep, $1 \%$ L-Glut, $5 \mathrm{ng} / \mathrm{ml} \mathrm{IL-6,} 50 \mathrm{ng} / \mathrm{ml}$ SCF, and $10^{3} \mathrm{U} / \mathrm{ml} \mathrm{LIF.} \mathrm{The} \mathrm{cultures} \mathrm{were} \mathrm{grown} \mathrm{at} 37^{\circ} \mathrm{C}$ in $5 \% \mathrm{CO}_{2}$ for 7 days, and the wells were collected, counted, and prepared for FACS analysis (SAHA, $1 \mu \mathrm{M}$; depsipeptide,10 nM). 
DNA fiber labeling. Bone marrow was harvested from the femurs of WT or Vav-Cre:Hdac3 $3^{-/-}$mice and cultured in RPMI media supplemented with $10 \%$ FBS, $1 \%$ Pen-Strep, and 1\% L-Glut. The culture medium was supplemented with $100 \mathrm{uM} \mathrm{CldU}$ for 20 minutes, and the cells were washed with PBS and then resuspended in culture medium containing $50 \mu \mathrm{M}$ IdU for $20 \mathrm{~min}$ utes. Stem and progenitor cells were then FACS sorted from 4 to $7 \times 10^{8}$ cells, and approximately 1,000 cell equivalents were spotted on slides. Three slides were prepared for each sample, and biological replicates were performed. DNA was fixed using a 3:1 mix of methanol/acetic acid and the slides were air dried. The incorporated thymidine analogs were detected on the spread fibers using anti-IdU (green) and anti-CldU (red) (goat anti-rat IgG Alexa Fluor 594 [CldU] and goat anti-mouse Alexa Fluor 488 [IdU] $1: 400$ in $10 \%$ goat serum/PBST; Invitrogen A- 11077 and A-11029) and performed as described (59), then photographed at $\times 1,000$ original magnification. At least 100 fibers were analyzed for each sample and a Mann-Whitney nonparametric $U$ test was used to test statistical significance.

Statistics. Statistical significance was determined by a 2 -tailed parametric Student's $t$ test (GraphPad Prism software). A $P$ value less than 0.05 was considered significant. Data are shown as the mean \pm SEM unless otherwise indicated in the legend.

Study approval. The present studies using mice were reviewed and approved by the Vanderbilt Institutional Animal Care and Use Committee of Vanderbilt University.

\section{Acknowledgments}

We thank all the members of Hiebert laboratory for helpful discussions, reagents and advice. We thank the Vanderbilt Cell Imag- ing, Translational Pathology, Flow Cytometry, and VANTAGE Shared Resources for services and support. This work was supported by the T.J. Martell Foundation; the Robert J. Kleberg, Jr. and Helen C. Kleberg Foundation; NIH grants (RO1-CA109355, RO1-CA164605, and R01-CA64140); and core services performed through the Vanderbilt Digestive Disease Research grant (NIDDK P30DK58404) and the Vanderbilt-Ingram Cancer Center support grant (NCI P30CA68485). A.R. Summers (5F32HL090259), A. Hunt (F30HL093993), and S. Bhaskara (1F32CA138091) were supported by fellowships from the NIH, and C.E. Wells, S. Bhaskara, and K.R. Stengel were supported by grant 5 T32 CA009582-25.

Received for publication July 3, 2012, and accepted in revised form April 16, 2013.

Address correspondence to: Scott W. Hiebert, Department of Biochemistry, 512 Preston Research Building, Vanderbilt University School of Medicine, 2220 Pierce Ave., Nashville, Tennessee 37232, USA. Phone: 615.936.3582; Fax: 615.936.1790; E-mail: scott. hiebert@vanderbilt.edu.

Srividya Bhaskara's present address is: Department of Radiation Oncology, Huntsman Cancer Center, University of Utah School of Medicine, Salt Lake City, Utah, USA.

Alyssa R. Summers' present address is: Department of Biology, Sewanee, The University of the South, Sewanee, Tennessee, USA.
1. Herman D, Jenssen K, Burnett R, Soragni E, Perlman SL, Gottesfeld JM. Histone deacetylase inhibitors reverse gene silencing in Friedreich's ataxia. Nat Chem Biol. 2006;2(10):551-558.

2. Rai M, et al. HDAC inhibitors correct frataxin deficiency in a Friedreich ataxia mouse model. PLoS One. 2008;3(4):e1958.

3. Thomas EA, et al. The HDAC inhibitor $4 \mathrm{~b}$ ameliorates the disease phenotype and transcriptional abnormalities in Huntington's disease transgenic mice. Proc Natl Acad Sci U S A. 2008;105(40):15564-15569.

4. $\mathrm{Xu} \mathrm{C}$, et al. Chemical probes identify a role for histone deacetylase 3 in Friedreich's ataxia gene silencing. Chem Biol. 2009;16(9):980-989.

5. Di Croce L. Chromatin modifying activity of leukaemia associated fusion proteins. Hum Mol Genet. 2005;1:R77-R84.

6. Buzzai M, Licht JD. New molecular concepts and targets in acute myeloid leukemia. Curr Opin Hematol. 2008;15(2):82-87.

7. Uribesalgo I, Di Croce L. Dynamics of epigenetic modifications in leukemia. Brief Funct Genomics. 2011;10(1):18-29.

8. Basso K, Dalla-Favera R. BCL6: master regulator of the germinal center reaction and key oncogene in B cell lymphomagenesis. Adv Immunol. 2010;105:193-210.

9. Richon VM, et al. A class of hybrid polar inducers of transformed cell differentiation inhibits histone deacetylases. Proc Natl Acad Sci U S A. 1998;95(6):3003-3007.

10. Xu WS, Parmigiani RB, Marks PA. Histone deacetylase inhibitors: molecular mechanisms of action. Oncogene. 2007;26(37):5541-5552.

11. Marks PA, Xu WS. Histone deacetylase inhibitors: Potential in cancer therapy. $J$ Cell Biochem. 2009;107(4):600-608

12. Zain J, O'Connor OA. Targeting histone deacetyalses in the treatment of B- and T-cell malignancies. Invest New Drugs. 2010;2(suppl 1):S58-S78.

13. Tan J, Cang S, Ma Y, Petrillo RL, Liu D. Novel histone deacetylase inhibitors in clinical trials as anti-cancer agents. J Hematol Oncol. 2010;3:5.

14. Lagger $\mathrm{G}$, et al. Essential function of histone deacetylase 1 in proliferation control and CDK inhibitor repression. EMBOJ. 2002;21(11):2672-2681.

15. Zimmermann S, et al. Reduced body size and decreased intestinal tumor rates in HDAC2-mutant mice. Cancer Res. 2007;67(19):9047-9054.

16. Jawerka $M$, et al. The specific role of histone deacetylase 2 in adult neurogenesis. Neuron Glia Biol. 2010;6(2):93-107.

17. Haberland M, Carrer M, Mokalled MH, Montgomery RL, Olson EN. Redundant control of adipogenesis by histone deacetylases 1 and 2.J Biol Chem. 2010;285(19):14663-14670.

18. Trivedi CM, et al. Hdac2 regulates the cardiac hypertrophic response by modulating Gsk3 beta activity. Nat Med. 2007;13(3):324-331.

19. Montgomery RL, et al. Histone deacetylases 1 and 2 redundantly regulate cardiac morphogenesis, growth, and contractility. Genes Dev. 2007;21(14):1790-1802.

20. Montgomery RL, Hsieh J, Barbosa AC, Richardson JA, Olson EN. Histone deacetylases 1 and 2 control the progression of neural precursors to neurons during brain development. Proc Natl Acad Sci U S A. 2009;106(19):7876-7881.

21. Haberland M, Johnson A, Mokalled MH, Montgomery RL, Olson EN. Genetic dissection of histone deacetylase requirement in tumor cells. Proc Natl Acad Sci U S A. 2009;106(19):7751-7755.

22. Yamaguchi $T$, et al. Histone deacetylases 1 and 2 act in concert to promote the G1-to-S progression. Genes Dev. 2010;24(5):455-469.

23. Furumai R, et al. FK228 (depsipeptide) as a natural prodrug that inhibits class I histone deacetylases. Cancer Res. 2002;62(17):4916-4921.

24. Witt O, Deubzer HE, Milde T, Oehme I. HDAC family: What are the cancer relevant targets? Cancer Lett. 2009;277(1):8-21.

25. Yang WM, Yao YL, Sun JM, Davie JR, Seto E. Isolation and characterization of cDNAs corresponding to an additional member of the human histone deacetylase gene family. J Biol Chem. 1997;272(44):28001-28007.

26. Nagy L, et al. Nuclear receptor repression mediated by a complex containing SMRT, $\mathrm{mSin} 3 \mathrm{~A}$, and histone deacetylase. Cell. 1997;89(3):373-380.

27. Heinzel T, et al. A complex containing N-CoR, $\mathrm{mSin} 3$ and histone deacetylase mediates transcriptional repression. Nature. 1997;387(6628):43-48.

28. Wen YD, et al. The histone deacetylase-3 complex contains nuclear receptor corepressors. Proc Natl Acad Sci U S A. 2000;97(13):7202-7207.

29. Li J, Wang J, Nawaz Z, Liu JM, Qin J, Wong J. Both corepressor proteins SMRT and N-CoR exist in large protein complexes containing HDAC3. EMBO J. 2000;19(16):4342-4350.

30. Guenther MG, Barak O, Lazar MA. The SMRT and $\mathrm{N}-\mathrm{CoR}$ corepressors are activating cofactors for histone deacetylase 3. Mol Cell Biol. 2001; 21(18):6091-6101.

31. Bhaskara S, et al. Deletion of histone deacetylase 3 reveals critical roles in $S$ phase progression and DNA damage control. Mol Cell. 2008;30(1):61-72.

32. Bhaskara S, et al. Hdac3 is essential for the maintenance of chromatin structure and genome stability. Cancer Cell. 2010;18(5):436-447.

33. Knutson SK, Chyla BJ, Amann JM, Bhaskara S, Huppert SS, Hiebert SW. Liver-specific deletion of histone deacetylase 3 disrupts metabolic transcriptional networks. EMBO J. 2008;27(7):1017-1028.

34. Montgomery RL, et al. Maintenance of cardiac energy metabolism by histone deacetylase 3 in mice. J Clin Invest. 2008;118(11):3588-3597.

35 . Feng D, et al. A circadian rhythm orchestrated by histone deacetylase 3 controls hepatic lipid metabolism. Science. 2011;331(6022):1315-1319.

36. McQuown SC, et al. HDAC3 is a critical negative regulator of long-term memory formation. J Neurosci. 2011;31(2):764-774.

37. Sun Z, et al. Diet-induced lethality due to loss of HDAC3 in heart and skeletal muscle. J Biol Chem. 2011;286(38):33301-33309.

38. Georgiades P, et al. VavCre transgenic mice: a tool 
for mutagenesis in hematopoietic and endothelial lineages. Genesis. 2002;34(4):251-256.

39. Adolfsson J, et al. Identification of Flt3+ lymphomyeloid stem cells lacking erythro-megakaryocytic potential a revised road map for adult blood lineage commitment. Cell. 2005;121(2):295-306.

40. Conti C, et al. Inhibition of histone deacetylase in cancer cells slows down replication forks, activates dormant origins, and induces DNA damage. Cancer Res. 2010;70(11):4470-4480.

41. Mao X, Fujiwara Y, Chapdelaine A, Yang H, Orkin $\mathrm{SH}$. Activation of EGFP expression by Cre-mediated excision in a new ROSA26 reporter mouse strain. Blood. 2001;97(1):324-326.

42. Lagasse E, Weissman IL. Flow cytometric identification of murine neutrophils and monocytes. J Immunol Methods. 1996;197(1-2):139-150.

43. Kiel MJ, Yilmaz OH, Iwashita T, Yilmaz OH, Terhorst C, Morrison SJ. SLAM family receptors distinguish hematopoietic stem and progenitor cells and reveal endothelial niches for stem cells. Cell. 2005;121(7):1109-1121.

44. Yilmaz OH, Kiel MJ, Morrison SJ. SLAM family markers are conserved among hematopoietic stem cells from old and reconstituted mice and markedly increase their purity. Blood. 2006;107(3):924-930.

45. Zhang $B$, et al. Altered microenvironmental regulation of leukemic and normal stem cells in chronic myelogenous leukemia. Cancer Cell.
2012;21(4):577-592.

46. Mansson R, et al. Molecular evidence for hierarchical transcriptional lineage priming in fetal and adult stem cells and multipotent progenitors. Immunity. 2007;26(4):407-419.

47. Broske AM, et al. DNA methylation protects hematopoietic stem cell multipotency from myeloerythroid restriction. Nat Genet. 2009;41(11):1207-1215.

48. Ng SY, Yoshida T, Zhang J, Georgopoulos K Genome-wide lineage-specific transcriptional networks underscore Ikaros-dependent lymphoid priming in hematopoietic stem cells. Immunity. 2009;30(4):493-507.

49. Pronk CJ, et al. Elucidation of the phenotypic, functional, and molecular topography of a myeloerythroid progenitor cell hierarchy. Cell Stem Cell. 2007;1(4):428-442.

50. Na Nakorn T, Traver D, Weissman IL, Akashi K. Myeloerythroid-restricted progenitors are sufficient to confer radioprotection and provide the majority of day $8 \mathrm{CFU}-\mathrm{S}$. J Clin Invest. 2002;109(12):1579-1585.

51. Schlegelberger B, Gohring G, Thol F, Heuser M Update on cytogenetic and molecular changes in myelodysplastic syndromes. Leuk Lymphoma. 2012;53(4):525-536.

52. Sirbu BM, Couch FB, Feigerle JT, Bhaskara S, Hiebert SW, Cortez D. Analysis of protein dynamics at active, stalled, and collapsed replication forks. Genes Dev. 2011;25(12):1320-1327.

53. Kawamoto H, Wada H, Katsura Y. A revised scheme for developmental pathways of hematopoietic cells: the myeloid-based model. Int Immunol. 2010;22(2):65-70.

54. Buza-Vidas N, Cheng M, Duarte S, Charoudeh HN, Jacobsen SE, Sitnicka E. FLT3 receptor and ligand are dispensable for maintenance and posttransplantation expansion of mouse hematopoietic stem cells. Blood. 2009;113(15):3453-3460.

55. Mann BS, Johnson JR, Cohen MH, Justice R, Pazdur R. FDA approval summary: vorinostat for treatment of advanced primary cutaneous T-cell lymphoma. Oncologist. 2007;12(10):1247-1252.

56 . Bishton MJ, et al. Deciphering the molecular and biologic processes that mediate histone deacetylase inhibitor-induced thrombocytopenia. Blood. 2011;117(13):3658-3668

57. Whittaker SJ, et al. Final results from a multicenter, international, pivotal study of romidepsin in refractory cutaneous T-cell lymphoma. J Clin Oncol. 2010;28(29):4485-4491.

58. Hug H, Costas M, Staeheli P, Aebi M, Weissmann C. Organization of the murine $\mathrm{Mx}$ gene and characterization of its interferon- and virus-inducible promoter. Mol Cell Biol. 1988;8(8):3065-3079.

59. Dorn ES, Chastain PD. Analysis of re-replication from deregulated origin licensing by DNA fiber spreading. Nucleic Acids Res. 2009;37(1):60-69. 